\title{
Diffraction and Forward Physics in ATLAS: results and perspectives
}

\author{
M. Bruschi ${ }^{1, a}$ on behalf of the ATLAS collaboration \\ ${ }^{1}$ I.N.F.N. Sezione di Bologna (ITALY)
}

\begin{abstract}
The present and future potential of ATLAS for diffraction and forward physics is presented. As recent results the rapidity gap cross section and elastic and total $p p$ cross sections are reported. The upgrade project AFP is presented and it is shown how it will complement the ALFA acceptance for diffractive physics in measurements taken with $\beta^{*}=90 \mathrm{~m}$ LHC beam optics. Moreover, the AFP detector will guarantee good acceptance on diffractive events also during normal running conditions allowing to improve the ATLAS detector performances. If in addition, a high luminosity program will be feasible, AFP might be fundamental for potential discoveries with extra dimensions being one example.
\end{abstract}

\section{Introduction}

Starting at the interaction point(IP) and proceeding along the beam line, there are the following forward detectors (FWD) in ATLAS :

- LUCID (LUminosity Cerenkov Integrating Detector), placed symmetrically at $17 \mathrm{~m}$ from the IP for the measurement of relative and absolute luminosity;

- ZDC (Zero Degree Calorimeter), placed symmetrically in the neutral absorber at about $140 \mathrm{~m}$ from the IP, for measurement of neutrons or other neutral particles produced in $p p$ or heavy ions (HI) interactions;

- ALFA (Absolute Luminosity For ATLAS), formed by two vertical RP stations per side, placed at about $240 \mathrm{~m}$ from the IP whose main goal is the measurement of the total cross section and luminosity.

The description of ATLAS and its FWD can be found in [1]. Besides these detectors, which are already installed and taking data, ATLAS foresees an additional upgrade project [2] named AFP (ATLAS Forward Proton) formed by 2 horizontal RP stations per side placed at $210 \mathrm{~m}$ with respect to the IP. The purpose of the FWD is to complement the ATLAS program by detecting particles that are produced at high rapidity and can not be studied with the central detectors.

The importance to instrument the forward part of ATLAS can be understood by inspecting the two plots reported in Fig. 1. The plot on the left shows the flow of the particles produced as a function of rapidity. The vertical dotted lines indicate the limit for reconstruction in the central detector set by the ATLAS tracker $( \pm 2.5$ rapidity units). It can be appreciated that about $40 \%$ of the tracks are produced out of the central detector acceptance. The

\footnotetext{
ae-mail: Marco.Bruschi@CERN.CH
}
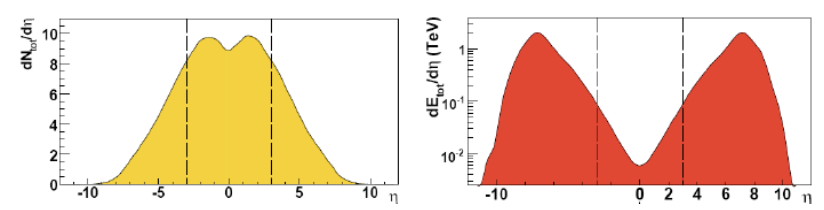

Figure 1. Particle flow (left) and energy flow (right) as a function of rapidity as obtained by DPMJET generator for $p p$ interactions at $\sqrt{s}=14 \mathrm{TeV}$

right plots in Fig. 1 shows instead the energy flow distribution as a function of the pseudo-rapidity, $\eta$. In this case it can be estimated that the energy released in the central detector is actually a very small fraction of the total energy produced. As a consequence, it is worth to instrument with detectors the forward part of ATLAS in order to better define the characteristics of the $p p$ interactions.

The outline of the document is the following. In Section 2 a measurement of the differential inelastic cross section as a function of the size of the rapidity gap will be described. This is an example of a measurement sensitive to diffractive physics using only the central detector. In Section 3 some details will be given about a study performed with the ALFA detector i.e. measurements at $\sqrt{s}=7 \mathrm{TeV}$ of the elastic differential cross section and the total cross section. In Section 4 the future perspectives using ALFA and AFP will be dicussed. Section 5 will finally present the future plans of measurements at low and medium number of interactions per bunch crossing $(\mu)$, respectively.

\section{Diffractive processes at ATLAS}

In Fig. 2 the typical classification of diffractive events in single diffractive (SD), double diffractive (DD), central diffractive $(\mathrm{CD})$ and elastic is reported. An indicative evaluation of the cross section for each topology at 


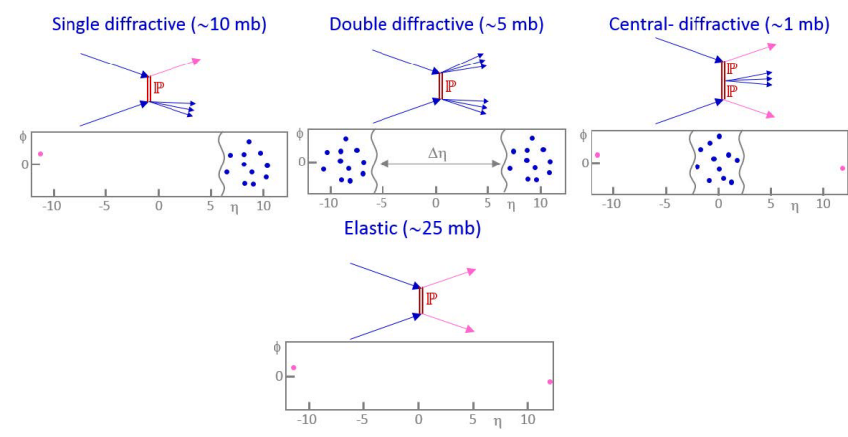

Figure 2. Classification of minimum bias processes. Their topology in the central and forward detectors is reported in the lower part of each class (azimuthal angle $\phi$ vs. rapidity $\eta$ ). In the pictorial view of the event, the pink dots represent the diffractively scattered proton(s), while the blue dots are the products of the diffractive $p p$ interaction.

$\sqrt{s}=14 \mathrm{TeV}$ is also reported. The diffractive and elastic events are about $40 \%$ of the LHC $p p$ collisions. Theoretically diffraction is referred to as a process where the quantum numbers of the vacuum are exchanged, or in the Regge theory, as a process with a $t$-channel exchange of a Pomeron. Experimentally, the presence of large gaps is a signature for diffraction, a common feature of all the event topology reported in Fig. 2. It has to be noted that ATLAS, with the forward calorimeter has an $\eta<4.9$ rapidity coverage which doesn't make it possible to access diffractive system mass less than $10 \mathrm{GeV}$. The indirect measurement of $\xi$ (which is the fractional longitudinal momentum loss of the protons) via the energy detected in the calorimeter is not very precise due to invisible energy and it works only in a limited region of $\xi$. Moreover no measurement of the Mandelstam variable $t$ of the proton is possible with the central detector. As a consequence, proton tagging is the only way for a more detailed probing of diffraction.

As an example to show the importance to tag protons in order to select diffractive events, the cross section as a function of the size of the rapidity gap as measured by the ATLAS central detector is considered [3]. In this analysis, the forward rapidity gaps $\left(\Delta_{\eta}^{F}\right)$ are defined as the larger forward rapidity gap region on detector edge devoid of particles having transverse momentum $P_{T}>200 \mathrm{MeV}$. In the left plot of Fig. 3 the inelastic cross section $\frac{d \sigma}{d \eta}$ is reported as a function of the gap size. The black dots are the measured data and the no diffractive (ND), SD and DD contribution of the Monte Carlo (MC) generator Pythia 8 [4] is reported superimposed. The other two plots (center and right of Fig. 3) report the same quantities for other MC generators, Pythia 6 and Phojet [5] respectively. A tagging of the proton would allow to better separate the different diffractive contributions.

The measured cross section is about $1 \mathrm{mb}$ per unit of gap size for a forward rapidity gap greater than 3 units. From all the plots it can be observed that the default Phojet and Pythia do not describe the rise of the cross section observed for $\Delta_{\eta}^{F}>5$. As shown in the plot reported in Fig. 4, the data are compared with a modified version
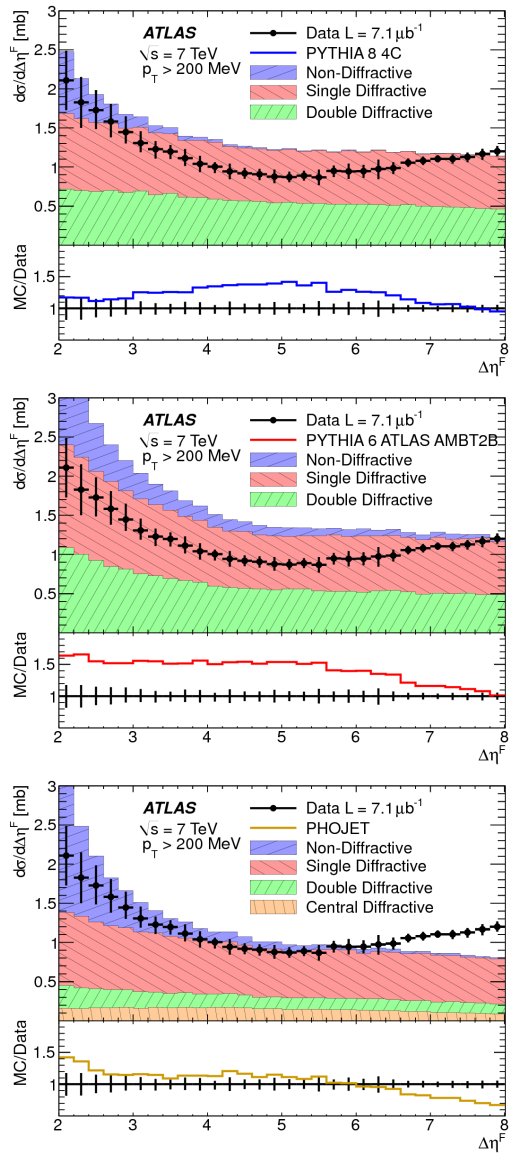

Figure 3. The black dots represent the measured inelastic cross section differential in forward gap size $\Delta_{\eta^{F}}$ for particles with $P_{t}>200 \mathrm{MeV}$ and $\Delta_{\eta^{F}}>2$. The error bars indicate the total uncertainties. The colored areas report the MC predictions of the generators Pythia 8 (left), Pythia 6 (center) and Phojet (right) for ND, SD and DD (also CD for Phojet) contributions [3].

of the Pythia 8 model with the Donnachie-Landshoff [6] Pomeron flux, in which the Pomeron intercept $\alpha_{P}(0)$ is determined from fits to the data in the region $6<\Delta_{\eta}^{F}<8$. The observed rise can be interpreted from a triple Pomeron contribution with a Pomeron intercept $\alpha_{P}(0)>1$. The slope is very sensitive to the precise value of $\alpha_{P}(0)$.

\section{Elastic and Total Cross Section Measurement}

In the following the result of the total cross section measurement at $\sqrt{s}=7 \mathrm{TeV}$ using the ATLAS-ALFA detector will be described [7]. The ALFA detector consists of $4+4$ vertical pots detectors symmetrically placed at about $240 \mathrm{~m}$ from the ATLAS IP. In Fig. 5 the topology of an elastic event is reported i.e.two back to back protons which traverse the ALFA stations. The elastic scattering is measured in a dedicated run of the LHC with a special high $\beta^{*}=90 \mathrm{~m}$ optics. The differential elastic cross section is used to estimate the total cross section as prescribed by the optical theorem:

$$
\sigma_{t o t} \propto 4 \pi \cdot \operatorname{I} m\left(f_{e l}\right)_{t \rightarrow 0}
$$




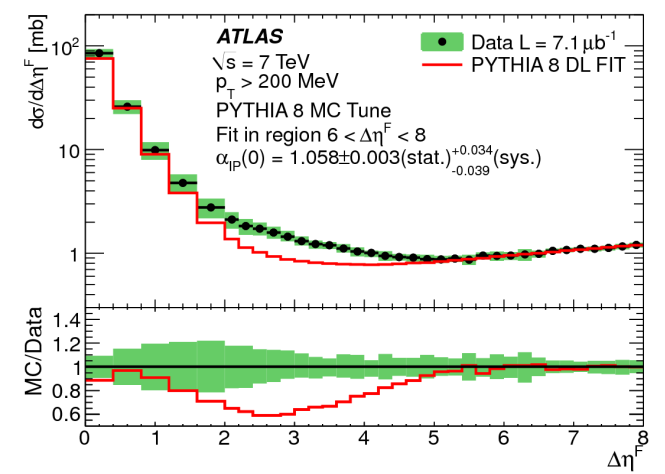

Figure 4. Inelastic cross section differential in forward gap size $\Delta_{\eta^{F}}$ for particles with $P_{t}>200 \mathrm{MeV}$. The data are compared with a modified version of the Pythia 8 model with the Donnachie-Landshoff Pomeron flux, in which the Pomeron intercept $\alpha_{P}(0)$ is determined from fits to the data in the region $6<\Delta_{\eta}^{F}<8[3]$

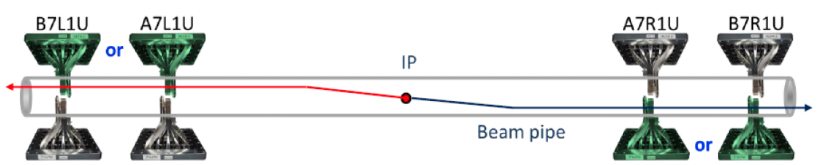

Figure 5. Pictorial view of a typical elastic event as detected by ALFA.

where $\sigma_{\text {tot }}$ is the total cross section, $f_{e l}$ is the elastic scattering amplitude and $t$ is the Mandelstam variable. Therefore, from a measurement of the elastic cross section differential in the $t$-variable and an extrapolation to $t=0$, the total cross section can be determined. Fig. 6 reports the differential cross section $\frac{d \sigma}{d t}$ as a function of the $t$ parameter. The details of the theoretical assumptions used for the fit of the elastic data can be found in [7]. The fit results gives a total cross section value of $95.4 \pm 1.3 \mathrm{mb}$ and a nuclear slope parameter $B$ of $19.73 \pm 0.24 \mathrm{GeV}^{-2}$ with the errors being the combination in quadrature of the experimental plus statistical errors. The lower part of the plot reports the percentage deviation of the fit with respect to the data showing superimposed the total error of the measurement and the statistical ones. The fit includes experimental systematic uncertainties in the $\chi^{2}$. The fit quality is good with the normalized $\chi_{N}^{2} \sim 0.46$. The fit range is set for $0.01<t<0.1 \mathrm{GeV}^{-2}$, where possible deviations from exponential form are small. Further uncertainty arise from the extrapolation of $t$ to zero, probed by a variation of the fit range upper limit from $0.1 \mathrm{GeV}^{-2}$ to $0.15 \mathrm{GeV}^{-2}$ and lower limit from $0.01 \mathrm{GeV}^{-2}$ to $0.058 \mathrm{GeV}^{-2}$. The extrapolation error on $\sigma_{\text {tot }}$ is $0.4 \mathrm{mb}$ and on the nuclear slope $B$ is $0.17 \mathrm{GeV}^{-2}$. The main sources of systematic experimental uncertainty for $\sigma_{t o t}$ are luminosity, $\sim 2.3 \%$, and the uncertainty on the beam energy which is $\sim 0.65 \%$. In Table 1 the breakdown of the total error for $\sigma_{t o t}$ and $B$ are reported in the statistical, experimental and extrapolation parts.

The next step for the ALFA detector will be to measure the differential elastic cross section and $\sigma_{\text {tot }}$ at $\sqrt{s}=$

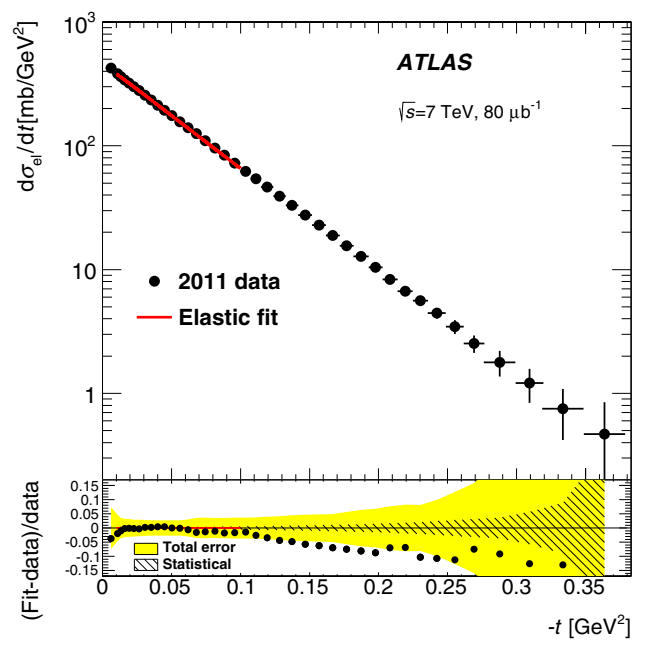

Figure 6. A fit of the theoretical prediction with $\sigma_{\text {tot }}$ and the nuclear slope $B$ as free parameters to the differential elastic cross section. In the lower panel the points represent the normalized difference between fit and data, the yellow area represents the total experimental uncertainty and the hatched area the statistical component. The red line indicates the fit range, the fit result is extrapolated in the lower panel outside the fit range [7].

\begin{tabular}{lcc}
\hline \hline & $\sigma_{\text {tot }}[\mathrm{mb}]$ & $B\left[\mathrm{GeV}^{-2}\right]$ \\
\hline Central result & 95.35 & 19.73 \\
Statistical error & 0.38 & 0.14 \\
Experimental error & 1.25 & 0.19 \\
Extrapolation error & 0.37 & 0.17 \\
\hline Total error & 1.36 & 0.35 \\
\hline
\end{tabular}

Table 1. Breakdown of the total error for $\sigma_{t o t}$ and $B$.

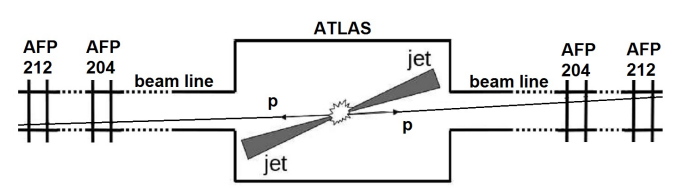

Figure 7. Sketch of the AFP detector.

$13 \mathrm{TeV}$. At a later stage the aim is to run with a $\beta^{*} \sim$ $2.5 \mathrm{~km}$ beam optics which will allow to reach the Coulomb nuclear interaction region (CNI), thus reducing the uncertainty of extrapolation to $t=0$ and providing a total cross section measurement not needing the luminosity as external parameter.

\section{Future perspectives: ALFA and AFP}

The AFP detector will consist of $2+2$ horizontal RP stations placed symmetrically at 204 and $212 \mathrm{~m}$ from the IP (see sketch reported in Fig. 7). Each two stations will allow to precisely define the position and direction of the 
proton trajectory. For events with two opposite protons in the final state, AFP will provide, independently from ATLAS central detector, the mass of the central event via the relation $M_{X}^{2}=s \xi_{1} \xi_{2}$ where $M_{X}$ is the mass of the central object produced, $s$ is the centre of mass energy squared, $\xi_{1}$ the fractional momentum loss of one proton and $\xi_{2}$ the fractional momentum loss of the other proton, respectively. This information can be used in a powerful exclusivity cut that will help to reduce the background in many measurements of central exclusive production (CEP) and will be decisive for some of them like the $\gamma \gamma$ final state (described in Section 5).

The first station at $204 \mathrm{~m}$ will host tracking detectors composed of the same 3D silicon sensors used in a part of the ATLAS insertable B layer [8], readout by FE I4 chips. The angular resolution will be at the level of $\mu \mathrm{rad}$ for proton tracks reconstruction. The second station at $212 \mathrm{~m}$ will contain both tracking detectors and a timing detector. The timing system should perform at $10 \mathrm{ps}$ or better time resolution (corresponding to about $2.1 \mathrm{~mm}$ precision on the reconstructed vertex in $z$ ) for proton pile-up background rejection. The baseline technology for timing consists in L-shaped quartz bars readout by fast micro channel plate photomultipliers, fast amplifiers, constant fraction discriminator and an HPTDC [9] based readout card. In parallel to this, there is an intense R\&D for Silicon based or Diamonds detectors readout by the SAMPIC [10] chip.

In Fig. 8 the results of beam optics simulation using the simulation code MAD-X [11] and MC generator level for the $\xi$ vs $p_{T}$ acceptance plots for AFP (upper plots) and ALFA (lower plots) is reported. The acceptance is evaluated for a $\beta^{*}=0.5 \mathrm{~m}$ (left plots) and a $\beta^{*}=90 \mathrm{~m}$ (right plots) beam optics. Low $\beta^{*}$ is associated with high luminosity while a high $\beta^{*}$ means low luminosity. The plots show that AFP has $\sim 100 \%$ acceptance at small $t$ while the $\xi$ range depends on the beam optics. ALFA has instead limited acceptance in $t$, broad acceptance in $\xi$ and good acceptance for elastics. Moreover, the ALFA detector can only be operated at very low luminosity and thus ALFA and AFP can play a complementary role for diffractive physics in ATLAS.

The whole forward region inclusive of the AFP detectors has been implemented in the ATLAS official MC code ATHENA [12]. Therefore performances of the detectors are available using the full simulation and also some physics channels have been studied, like the double Pomeron exchange (DPE) dijets. These studies have shown that acceptance of AFP is typically in the range $0.015<\xi<0.15$.

\section{Program of measurements at low and low/medium luminosity}

At the beginning of year 2014, ATLAS approved a physics program to be performed with AFP in special beam conditions at very low and low/medium intensity. The very low luminosity program $(\mu<0.05, \mu$ being the average number of interactions per bunch crossing) will study typical aspects of soft QCD like rapidity gap cross section,
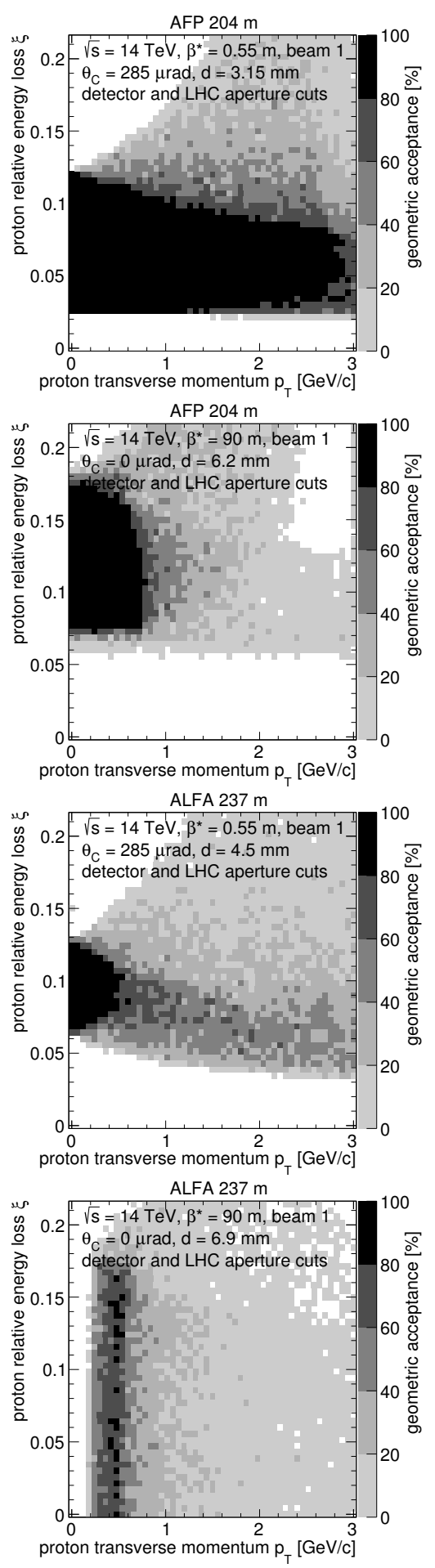

Figure 8. $\xi$ vs $p_{T}$ acceptance plots for AFP (upper plots) and ALFA (lower plots). The acceptance is evaluated at $\beta^{*}=0.5 \mathrm{~m}$ (left plots) and $\beta^{*}=90 \mathrm{~m}$ (right plots) LHC beam optics.

underlying event and multi-parton interactions. With its ability to tag diffractively scattered protons, AFP will be decisive in improving the selection of SD and DD events and shed light on the interpretation of the data in terms of the diffractive processes .

Besides the very low luminosity program, AFP and ALFA are planning to take data at higher luminosity for 

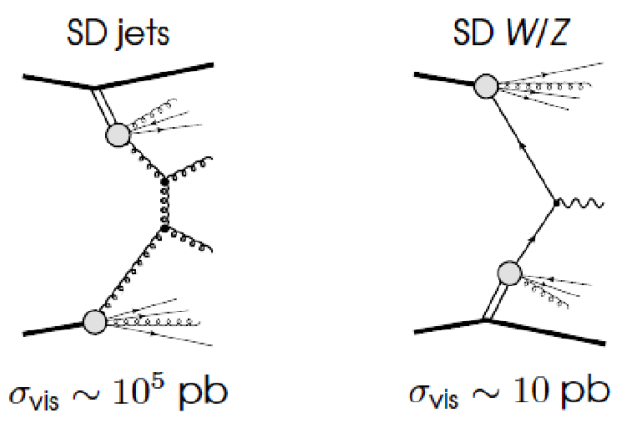

Figure 9. Typical hard diffractive processes to be studied at LHC and their cross sections $\sigma_{v i s}$ at $\sqrt{s}=13 \mathrm{TeV}$.

$0.5<\mu<3$. In 100 hours $(\sim 1$ week) of data taking this would allow to collect $\sim 40 \div 200 p b^{-1}$ and study hard diffractive processes (see Fig. 9).

One of the processes that can be studied is SD vector boson or jet production. With this set of data it will be possible for example to study the rapidity gap survival probability $S^{2}$ [13]. In the case of the single diffractive $W$ production, the charge asymmetry will allow constraining the flavor composition of the Pomeron. Running for the order of 100 hours at $\mu \sim 1$ will allow to get a significance (defined as $\frac{S}{\sqrt{S+B}}, S$ being the signal and $B$ the background of the measurement) above 20. Another case of interest is the DPE jets production. In this case the two protons exchange two colorless objects (Pomerons) and leave the IP intact. The two Pomerons produce centrally a dijet and also other remnants (see rightmost diagram of Fig. 9). With this class of events it will be possible to study the gluon contents of the Pomeron and the $\beta$ dependence of the Pomeron structure, $\beta$ being the momentum fraction of the Pomeron carried by the interacting parton inside the Pomeron. In this case, running at $\mu \sim 1$ for $\sim 100$ hours will allow to get a significance above 1000 for a jet $p_{T}>20 \mathrm{GeV}$ and significance of about 50 for a jet $p_{T}>100 \mathrm{GeV}$.

Another important diagram is the CEP in which there is the exchange of two gluons with a third gluon contributing to produce a colorless object. The two protons are intact, and the central object is produced without remnants. In this case the mass reconstructed in AFP is equal to the mass reconstructed in the central detector, and this can be used as a powerful cut to reject background events. The exclusive jets production will allow to study the unintegrated gluon distributions.

The diffractive production of gamma+jet, at the LHC energy scale, will allow to distinguish between diffraction mechanisms as it can be appreciated in the simulation reported in Fig. 10 [14]. In the plot the ratio of the gamma+jet cross section to the dijet cross section is reported as a function of the measured mass of the central object. It can be seen that the soft color interaction (SCI) model predicts a constant ratio all over the mass range, while the DPE model has a different trend. Moreover, the ratio in this case depends on the quark $u / d$ ratio in the

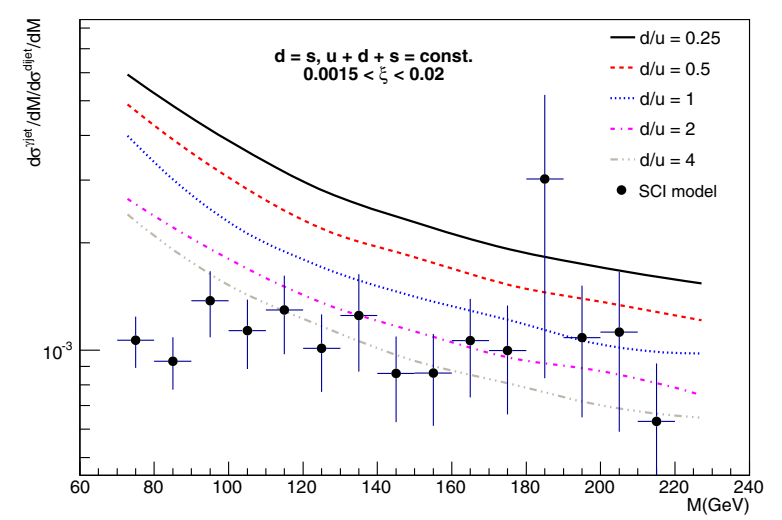

Figure 10. Simulation results of the ratio of the gamma+jet cross section to the dijet cross section as a function of the central mass. The ratio is evaluated for different Pomeron $d / u$ values and for the SCI model predictions [14].

Pomeron. One day at low luminosity (about $10 \mathrm{pb}^{-1}$ ) will be enough to perform this measurement. The measurement of the dijet cross section will also allow to check if the Pomeron is indeed the same at $e p$ or $p p$ colliders, which is a fundamental theoretical question.

Finally, SD or DPE events of the type jet-gap-jet will probe the Balitsky-Fadin-Kuraev-Lipatov (BFKL) [15] dynamics of the Pomeron. This can be studied by calculating the ratio of the DPE jet-gap-jet to the DPE dijets cross sections (suitably corrected for leading order and next to leading order effects) as a function of the leading jet $p_{T}$. It is important to note that the chosen ratio minimize systematics uncertainties since, for instance, the factors $S^{2}$ cancel. The needed luminosity to perform this measurement is $300 \mathrm{pb}^{-1}$. The theory predicts an almost constant ratio of 0.25 over all the leading jet $p_{T}$ range. Deviations from this trend will reveal aspects of the Pomeron dynamics.

Besides the low $\mu$ program approved by ATLAS for the run starting in year 2015, AFP might be a powerful tool also for discovery physics. This requires that the beam related background at normal optics condition (low $\beta^{*}$, high luminosity) is tolerable. Here photon exchange processes with $W W, Z Z$ and $\gamma \gamma$ final states can be accessed using the exclusivity cut from the AFP measured mass. Such measurements will allow to test quartic gauge couplings (QGC) and get insight in Beyond Standard Model (BSM) theories. Standalone simulations, in the $W W$ final state case, reported for instance in [2], have shown that the presence of AFP will allow to improve of about 4 order of magnitude the sensitivity reached by OPAL and one-two order of magnitudes the sensitivity reachable using only the ATLAS central detector collecting a statistics of $\sim 200 \mathrm{fb}^{-1}$. This will allow to reach coupling values expected in extradimension models.

Of particular relevance, in this set of measurements, is the case of $\gamma \gamma \gamma \gamma$ coupling, since simulations have shown that AFP allows to reach sensitivities required to test string theory and grand unification models. In contrast to mea- 
surements of the the other possible vector boson couplings, which require a time resolution of $10 \mathrm{ps}$ or better, the $\gamma \gamma$ final state will require only moderate timing resolution, due to the powerful exclusivity cut on the central $\gamma \gamma$ mass provided by AFP.

At moderately high or high luminosity it will also be possible to study exclusive jet production to verify QCD production models .

The moderately high or high luminosity program sketched above is still not approved by ATLAS and it will be reviewed when real data on background in normal running conditions will be available.

\section{Conclusions}

The ATLAS experiment produced and is producing important results for diffractive physics. Those results are expected to provide a valuable input for modeling and tuning of MC generators, to study mechanism of diffractive processes, mechanism of hadronization and confinement and for search for new QCD dynamics.

The quality of these studies will improve when the detector will be upgraded with AFP adding the capability to provide proton tagging. ALFA will continue its program of total cross section measurements at the new LHC energy and at higher $\beta^{*}$ (about $2.5 \mathrm{~km}$ ), thus reaching inside the CNI region. ALFA will also contribute to complement the AFP acceptance in the high $\beta^{*}$ - low luminosity physics program.

The hard diffractive program of AFP at low $\beta^{*}$ - high luminosity is indeed very interesting, but it will only be approved when data on the beam background in normal running conditions are available.

\section{Acknowledgement}

Warm thanks to Per Grafstrom and Christophe Royon for many useful discussions.

\section{References}

[1] ATLAS Collaboration, JINST 3, S08003 (2008)

[2] ATLAS Collaboration, CERN-LHCC-2011-012

[3] ATLAS Collaboration, Eur. Phys. j. C72 (2012) 1926

[4] T. Sjöstrand et al, CERN-LCGAPP-2007-04

[5] F.W. Bopp et al, http://arxiv.org/abs/hep-ph/9803437

[6] A. Donnachie, P. Landshoff, Nucl. Phys. B 244 (1984) 322

[7] ATLAS Collaboration, Nucl. Phys. Section B (2014) 486-548

[8] ATLAS Collaboration, CERN-LHCC-2010-013

[9] J. Pinfold, Act. Phys. Polon. B, Proc. Suppl., 4 (2011) 71

[10] E. Delagnes et al., Act. Phys. Polon., Proc. Suppl., 7 (2014) 685

[11] E. Forest, F.Schmidt and E. McIntosh, CERN-SL2002-044 (AP) KEK-report 2002-3

[12] ATLAS Collaboration, CERN-LHCC-2005-022

[13] CDF Collaboration, Phys. Rev. Lett. 84 (2000) 504

[14] C. Marquet, C. Royon, M. Saimpert, and D. Werder, arXiv:1306.4901 [hep-ph]

[15] L.N. Lipatov, Sov. J. Nucl. Phys. 23 (1976) 338 